\title{
An Alternative Strategy for the Use of a Low-Cost, Age- Hard enable Fe-Si-Ti Steel for Automotive Application
}

\author{
Bouaziz 0 ${ }^{1,2 *}$, P Barges $^{3}$ \\ ${ }^{1}$ Laboratoire d'Etude des Microstructures et de Mécanique des Matériaux (LEM3), CNRS, Université de Lorraine, Arts et Métier Paris Tech, \\ F 57000, Metz, France
}

${ }^{2}$ Laboratoire d'EXcellence DAMAS

${ }^{3}$ Arcelor Mittal Research, Voie Romaine-BP30320,57283 Maizières-lès-Metz Cedex, France

*Corresponding author: Bouaziz 0, Laboratoire d’Etude des Microstructures et de Mécanique des Matériaux (LEM3), CNRS, Université de Lorraine, Arts et Métier Paris Tech, F 57000, Metz, France

\begin{abstract}
For High Strength Low Alloy (HSLA) steels or for age-hard enable steels (miraging) the strengthening by precipitation is done before forming operation to increase the yield stress as much as possible. In this publication the advantages of a hardening thermal treatment after forming operation are investigated in a low-cost age-hard enable steel Fe-Si-Ti consistent with automotive application.
\end{abstract}

Keyword: Age-hard enable, Precipitation, Forming, Ductility

\section{Introduction}

The high strength low alloy (HSLA) steels are a group of low carbon steels with small amounts of alloying elements (as Ti, V, $\mathrm{Nb}$, etc.) to obtain a good combination of strength, toughness, and weldability [1-3]. By the addition of micro-alloying elements (less than 0.5 wt.\%), HSLA steels are strengthened by grain refinement

strengthening, solid solution strengthening and precipitation hardening [4-8]. Regarding automotive applications, the entire range of HSLA steels are suitable for structural components of cars, trucks, and trailers such as suspension systems, chassis, and reinforcement parts. HSLA steels offer good fatigue and impact strengths. Given these characteristics, this class of steels offers weight reduction for reinforcement and structural components [9-11]. Despite the interest of HSLA, the precipitation hardening is about $100 \mathrm{MPa}$. So the new targets concerning the $\mathrm{CO}_{2}$ emission of vehicles push the steel-makers to develop Advanced High Strength Steels (Dual-Phase, Transformation Induced Plasticity) hardened by multiphase microstructures containing 10 to $100 \%$ of martensite [12] which offer a better combination between strength and ductility for an acceptable cost Let's notice that the Ultimate Tensile Strength (UTS) increases more that the Yield Stress (YS).
YS is crucial for anti-intrusive aspect during a crash [12]. In the other side, because there is no phase transformation in Aluminum, age-hardening by precipitation is widely used in Aluminium alloys hardened by various additive elements [see [13] for a review]. The volume fraction of precipitates is several percent's, the hardening can be increased up to $400 \mathrm{MPa}$, but the ductility decreases rapidly.

In steel, there is only miraging steels which are strengthened by a massive precipitation of a martensitic matrix [see [14] for a review]. Despite the impressive YS (up to 2.5GPa), the uniform elongation is less than $1 \%$. Consequently, no forming operation is possible. In addition, the high contents of nickel (about 18\%), of molybdenum and of cobalt make these steels very expensive and so they are never used in automotive industry. On the contrary, in the 60 's authors investigated other kind of steels suitable to be strongly hardened by massive intermetallic precipitation without extracost $[15,16]$. Among the different investigated system, the Fe-Si-Ti alloys are the most promising. This is the reason why a series of publications has been dedicated to this system in the 70's by Jack\&al. [17-19]. Unfortunately, hardness and compression behaviour have been only reported and a lot of discussions concerned the nature of 
the precipitates $\left(\mathrm{Fe}_{2} \mathrm{Ti}, \mathrm{FeSi}\right.$, or $\left.\mathrm{Fe}_{2} \mathrm{SiTi}\right)$ have been reported. More recently a systematic study of precipitation kinetics in a $\mathrm{Fe}_{-2} .5 \% \mathrm{Si}$ $1 \%$ Ti alloy in the temperature range $723 \mathrm{~K}$ to $853 \mathrm{~K}\left(450^{\circ} \mathrm{C}\right.$ to $\left.580^{\circ} \mathrm{C}\right)$, combining complementary tools transmission electron microscopy (TEM), atom probe tomography (APT), and Small-Angle-Neutron Scattering (SANS)) have been carried out [20]. It has been shown that the Heusler phase $\mathrm{Fe}_{2} \mathrm{SiTi}$ dominates the precipitation process in the investigated time and temperature range, regardless of the details of the initial temperature history.

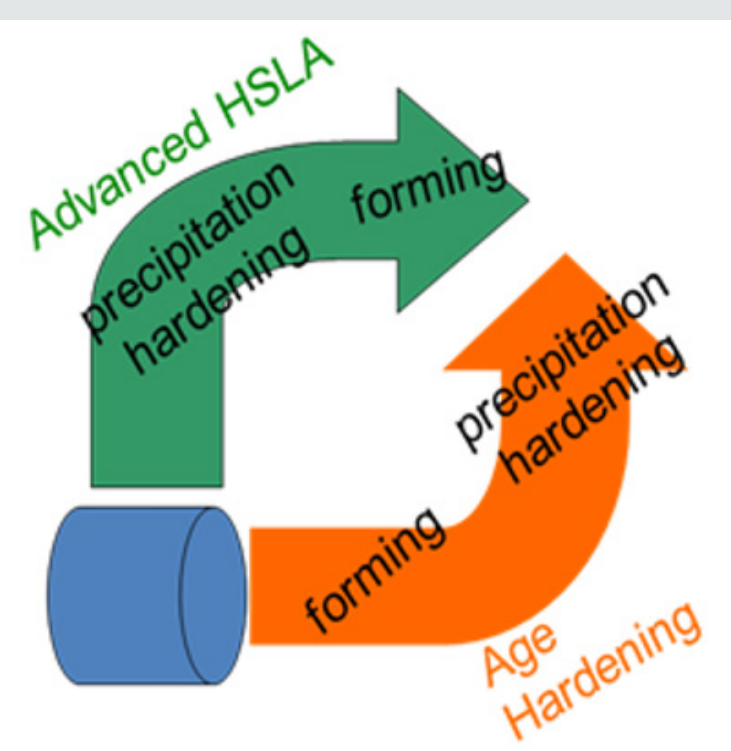

Figure 1: Summary of the different strategies for the use of precipitation hardened steels: The green arrow is the usual one, the orange is the strategy developed in this publication.

Considering that the ductility decreases regularly as a function of the hardening up to $1.2 \mathrm{GPa}$ and because it is targeted to obtain a steel suitable for deep drawing the strategy showed in (Figure 1). (orange arrow) has been investigated. The objective is to form a material as soft as possible and as ductile as possible and to obtain the hardening by a thermal treatment after forming. This publication presents the characterization of this way to manage the situation. It is noticed that as $\mathrm{Si}$ and Ti promote ferrite, there is no phase transformation whatever the thermal treatment. (Figure 1) Summary of the different strategies for the use of precipitation hardened steels: The green arrow is the usual one, the orange is the strategy developed in this publication.

\section{Results}

In order to assess for the first time in steel for automotive applications, the hardening have been chosen followed a thermal treatment at $500^{\circ} \mathrm{C}$ for two hours consistent with the kinetic determined by SANS [20]. As shown in (Figure 2). illustrating the tensile behavior of the steel consisting only in solid solution (i.e. only after recrystallization) is very ductile as for IF steels but with a higher yield stress due to solid solution hardening. After treatment and hardening of $300 \mathrm{MPa}$ is obtained with promising ductility. As shown in (Figure 3) the treatment has induced the expected massive precipitation of $\mathrm{Fe}_{2} \mathrm{SiTi}$ (3.8\% weight percent with a radius of $4 \mathrm{~nm}$ determined by TEM and SANS [20]). To assess the metallurgical route, the (Figure 4). shows that it is possible to severely bend the steel consisting only in solid solution without any defect up to an angle of $180^{\circ}$. Hardness trough the thickness has been measured before and after the thermal treatment (Figure 5). The value confirms the hardening by precipitation. It is noticed that tis precipitation hardening is not sensitive to the strain hardening induced by bending trough the thickness. Because the alloy is dedicated to automotive industry, the drawing must be investigated. This the reason why a cup obtained by deep drawing of $5 \mathrm{~cm}$ diameter has been manufactured using the steel consisting in solid solution without any problem as illustrated in (Figure 6). It confirms the very high ductility before heat treatment. One another problem in automotive industry is the increase in spring-back with an increase in strength. In addition, it is very difficult to predict or to model this phenomenon. As shown in (Figure 7). this aspect has been studied by a standard test base the forming of a hat-shaped part. It is highlighted that the spring-back during the treatment is weak. That is probably because there is no phase transformation during precipitation and so no internal stresses.

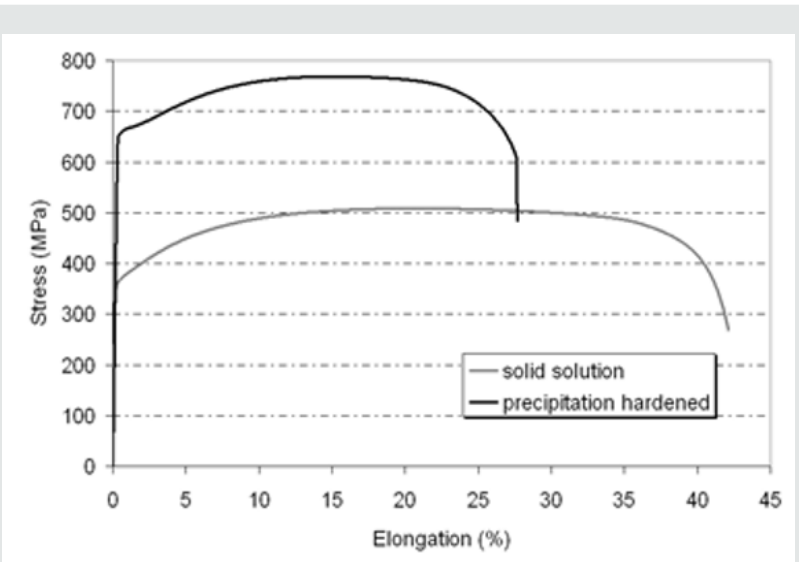

Figure 2: Tensile curves before and after the ageing treatment. 


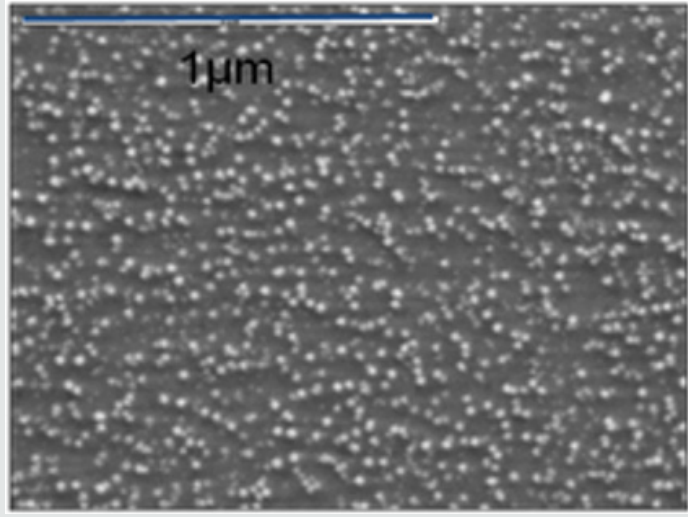

Figure 3: TEM micrography showing the massive precipitation of $\mathrm{Fe}_{2} \mathrm{SiTi}$ after 2 hours at $500^{\circ} \mathrm{C}$ (the composition have been determined by APT [20]).

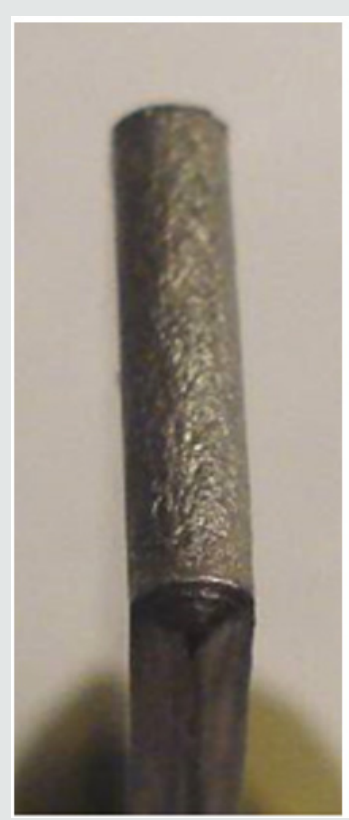

Figure 4: Fully bent specimen before heat treatment (bending angle of $180^{\circ}$ ).

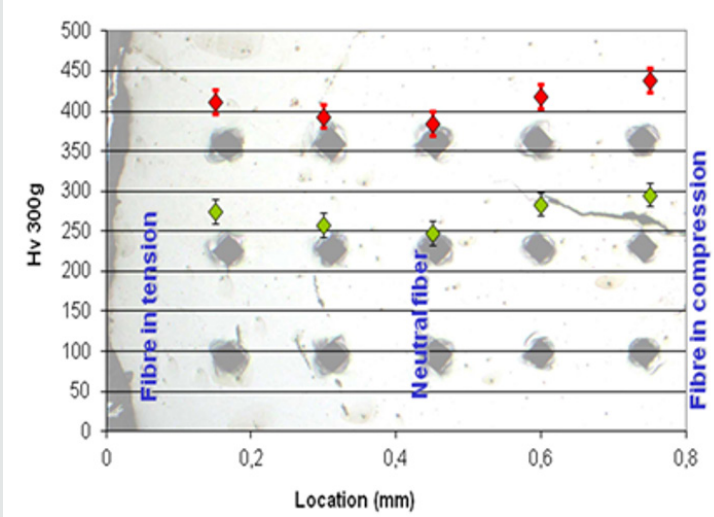

Figure 5: Hardness measurement trough the thickness of the fully bent specimen (i.e. angle of $180^{\circ}$ ) before and after heat treatment.

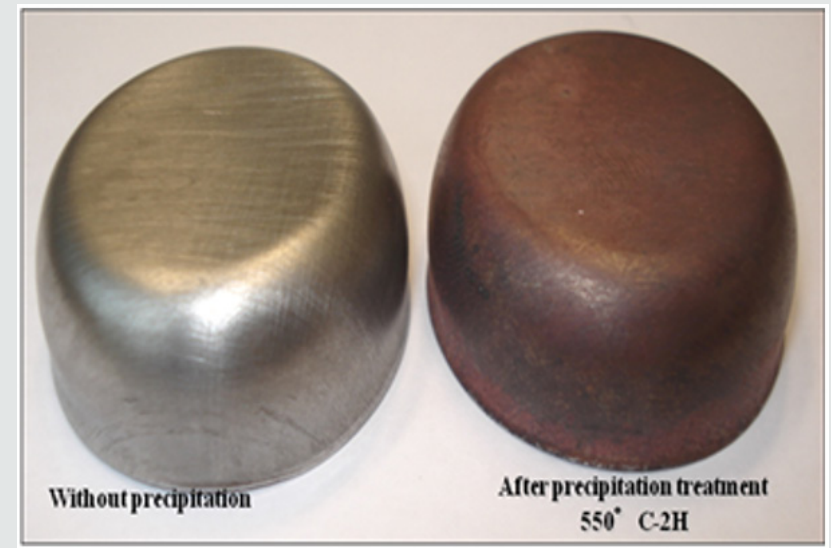

Figure 6: Cup drawing before the hardening heat treatment (5cm diameter).

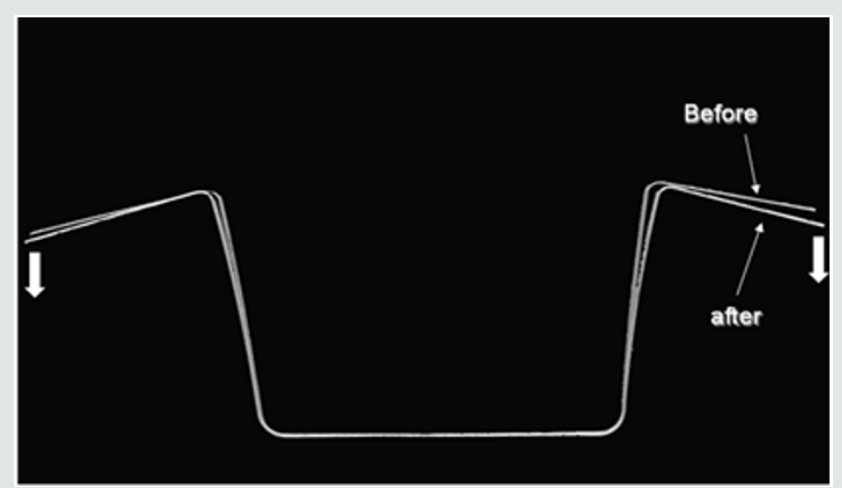

Figure 7: Evaluation of the effect of heat treatment on the spring-back.

\section{Conclusion}

For the first time in steel industry for automotive industry a low cost age-hard enable steel has been studied following a strategy based on forming operations before the heat treatment. The bending, the drawability and the spring-back have been investigated highlighting promising results. In addition, the alloy exhibits a cost acceptable for automotive industry. In the future crashworthiness and weldability should be assessed after heat treatment. One of the last advantages is that a lot of parts can be treated at the same time in a furnace usually dedicated to tempering treatment.

\section{References}

1. Porter LF, Repas PE (1982) The evolution of HSLA steels. JOM 34(4): 1421.

2. NJ Kim (1983) The physical metallurgy of HSLA linepipe steels-a review. JOM 35(4): 21-27.

3. Shao Y, Liu C, Yan Z, Li H, Liu Y (2018) Formation mechanism and control methods of acicular ferrite in HSLA steels: a review. J Mater Sci Technol 34(5): 737-744.

4. Kejian $\mathrm{H}$, Baker $\mathrm{T}$ (1996) Zr-containing precipitates in a $\mathrm{Ti} \mathrm{Nb}$ microalloyed HSLA steel containing $0.016 \mathrm{wt} . \% \mathrm{Zr}$ addition. Mater Sci Eng. A 215(1): 57-66. 
5. Rodrigues P, Pereloma E, Santos D (2000) Mechanical properties of an HSLA bainitic steel subjected to controlled rolling with accelerated cooling. Mater Sci Eng. A 283(1): 136-143.

6. Charleux M, Poole W, Militzer M, Deschamps A (2001) Precipitation behavior and its effect on strengthening of an HSLA-Nb/Ti steel. Metall Mater Trans. A 32(7): 1635-1647.

7. Park DB, Huh MY, Shim JH, Suh JY, Lee KH, Jung WS (2013) Strengthening mechanism of hot rolled $\mathrm{Ti}$ and $\mathrm{Nb}$ microalloyed HSLA steels containing Mo and W with various coiling temperature. Mater Sci Eng A 560: 528534.

8. Huang MX, He BB (2018) Alloy design by dislocation engineering. J Mater Sci Technol 34(3): 417-420.

9. https://automotive.arcelormittal.com/products/flat/HYTSS/HSLA

10. Todd M. Link, U.S. Steel, Private Communication.

11. https://bodybuilder.scania.com/trucks/en/home.html

12. Bouaziz O, Zurob H, Huang MX (2013) Driving Force and Logic of Development of Advanced High Strength Steels for Automotive Applications. steel research int. 1: 184.
13. Dursuna T, Soutisb C (2014) Recent developments in advanced aircraft aluminium alloys. Materials and Design 56: 862-871.

14. Sha W, Guo Z (2009) A volume in Woodhead Publishing Series in Metals and Surface Engineering.

15. Hénon JP, Waché C, Manenc J (1966) Etude de la précipitation dans les alliages Fe-Ti, Fe-Si-Ti, Fe-Ti-Ni, , Mémoires scientifiques revues métallurgiques, LXIII: 2.

16. Hobbs RM, Brammar IS (1968) Transactions of the Japan Institute of Metals 9.

17. Jack DH (1973) Invited review: Carbides and nitrides in steel Metal science Journal 4.

18. DH Jack, Honeycombe RWK (1972) Acta Metallurgica 20 (6): 787-796.

19. Jack DH, Guiu F (1975) Journal of Materials Science 10: 1161-1168.

20. Perrier M, Bouaziz O, Brechet Y, Danoix F, De Geuser F, et al. (2012) Metall Mater Trans A (43): 4999-5008.

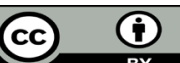

This work is licensed under Creative Commons Attribution 4.0 License

To Submit Your Article Click Here:

Submit Article
DOI: $10.32474 /$ MAMS.2020.03.000156

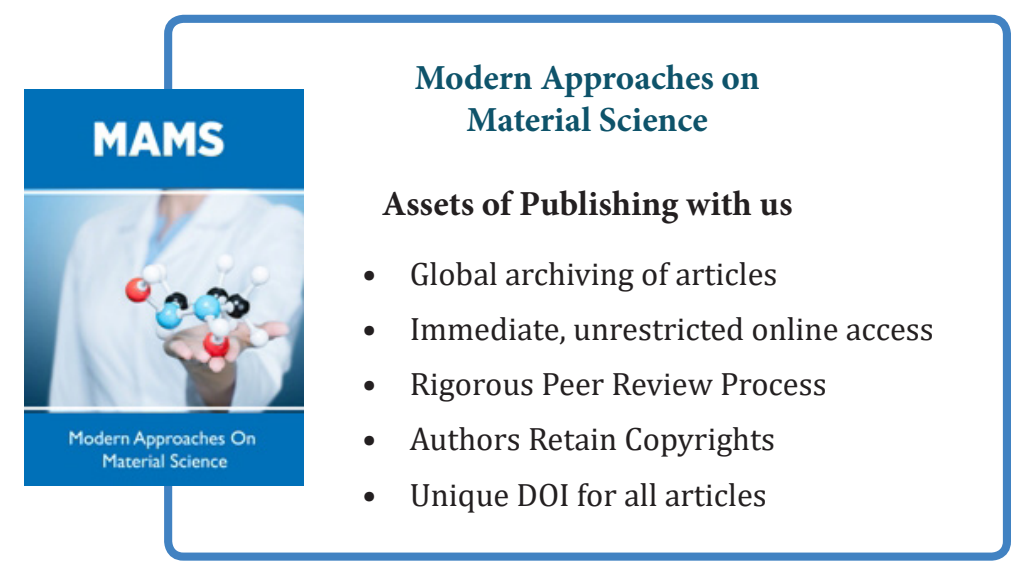

